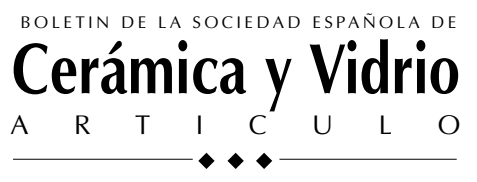

\title{
Estudio reológico de mezclas de porcelana reciclada para el moldeo por inyección
}

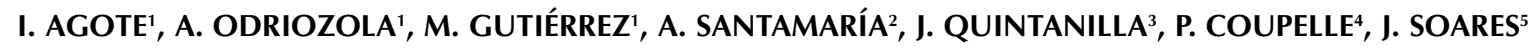 \\ 'Fundación INASMET, 20009 San Sebastián. \\ 2Departamento de Ciencia y Tecnología de Polímeros, Facultad de Ciencias Químicas, UVP/EHU, 20080, San Sebastián \\ ${ }^{3}$ Porcelanas del Bidasoa .20303 Irun . \\ ${ }^{4} \mathrm{KPCL}$ Avenue du Président-Kennedy. 87015 Limoges Cedes, Francia. \\ ${ }^{5}$ SOARMOLDES Praia da Granja. 4411 Valadares Codex., Portugal.
}

El objeto de este trabajo es el de investigar la posibilidad de poder reutilizar porcelana de desecho para el moldeo por inyección. Para ello se han estudiado cinco formulaciones conteniendo diferentes cantidades de porcelana reciclada. En ellas se han evaluado diversos parámetros significativos de flujo y se ha analizado su relación con el comportamiento en la inyección.

Palabras Clave: Moldeo por Inyección de Cerámicas, Reología, Porcelana.

Rheological study of waste porcelain mixtures for injection molding.

The aim of this work is to investigate the possibility of using waste porcelain for injection moulding. Five formulations of porcelain containing different amounts of recycled porcelain have been studied. Flow properties of these formulations have been analysed and related to injection behaviour.

Keywords: Ceramic Injection Moulding, Rheology, Hard Porcelain.

\section{1.- INTRODUCCION}

En estos tiempos en los que el mercado exige ser extremadamente competitivos, es de suma importancia reducir el precio del producto, sin alterar su calidad. El Moldeo por Inyección de Cerámicas (MIC) es una técnica que permite fabricar piezas de una geometría compleja a cotas finales que no requieren mecanizado y combina un alto volumen de producción de piezas con unas tolerancias dimensionales muy estrechas.

En el proceso de MIC el papel de la mezcla es muy importante. Las propiedades de flujo de ésta (reología) deben permitir un buen llenado de la cavidad de moldeo. En la preparación de las mezclas para la inyección, se intenta utilizar la mínima cantidad de ligante posible que permita un buen comportamiento reológico. Esto permite obtener altas densidades en verde y una menor contracción de la pieza durante el sinterizado.

El proceso de moldeo por inyección de cerámicas (MIC) representa una excelente alternativa para la producción de productos de la industria de vajilla de un modo más automatizado. Una característica de este proceso es que permite la reutilización de las piezas rechazadas de porcelana, reduciendo el coste del producto.

Este estudio trata de probar la posibilidad de reutilizar porcelana mediante la técnica del moldeo por inyección. Con objeto de evaluar el comportamiento de las mezclas de porcelana, se intenta establecer una relación entre las características del material en polvo y el comportamiento reológico de las mezclas así como predecir el comportamiento de la mezcla en la etapa de inyección mediante la utilización de los datos reológicos.

\section{2.- PROCEDIMIENTO EXPERIMENTAL}

\section{1- Materiales}

\subsection{1.- LA PORCELANA}

El material cerámico estudiado es porcelana dura (hard porcelain), la cual está compuesta principalmente por Caolín, Cuarzo y Feldespato en concentraciones relativas de 50, 25 y $25 \%$ respectivamente. Comparada con otras porcelanas, es muy dura y la temperatura de cocción es más alta: $1350^{\circ} \mathrm{C}$ $[1,2]$.

Se han utilizado dos tipos de porcelana:

a) Porcelana Virgen.

b) Porcelana reciclada.

Se han empleado dos fuentes de obtención de porcelana reciclada:

- Porcelana Bizcochada: Esta porcelana ha sido cocida a $900^{\circ} \mathrm{C}$. Es fácil de moler debido a que la etapa de cocción no se ha finalizado totalmente.

- Porcelana Calcinada: Esta porcelana ha sido cocida a $1350^{\circ} \mathrm{C}$. A esta temperatura el proceso de cocción ha sido completado. Esta porcelana es más difícil de moler.

Cinco formulaciones de porcelana fueron seleccionadas con objeto de determinar la influencia de la porcelana reciclada en la reología de las mezclas para el moldeo por inyección. Las características de estas formulaciones están descritas en la Tabla 1. Las formulaciones 1, 3 y 5 fueron seleccionadas con objeto de estudiar la influencia de la porcelana calcinada mezclada con la virgen. La formulación 5 es la que mayor contenido de porcelana reciclada tiene: $80 \%$ en peso. La formula- 
ción 2 estudia la influencia de la porcelana bizcochada mezclada con la virgen. La formulación 4 ha sido elegida como referencia y solamente está compuesta por porcelana virgen.

De las propiedades físicas descritas en la Tabla 1, el tamaño medio de partícula y la distribución de tamaños de partícula y superficie específica se han obtenido mediante el análisis de las muestras en un medidor de láser tipo "Malvern Instrumentes laser granulometer". Estas propiedades granulométricas son especialmente importantes para el proceso de moldeo por inyección. El grado de distribución de tamaños de partícula, ha sido determinado mediante el parámetro de pendiente $\left(S_{w}\right)$ y el grado de compactación.

Además del contenido de porcelana reciclada, existen otras diferencias en las formulaciones de porcelana:

Las formulaciones 1 y 3 tienen el mismo contenido de porcelana reciclada, pero diferente tiempo de molienda, lo que hace que el tamaño medio de partícula sea diferente.

Las formulaciones 1 y 2 tienen el mismo tamaño medio de partícula, pero diferente distribución de tamaños de partícula. Lo mismo ocurre entre las formulaciones 3 y 4.

Las formulaciones 4 y 5 tienen el mismo grado de compactación pero diferente tamaño medio de partícula.

TABLA 1: CARACTERÍSTICAS DE LAS FORMULACIONES DE PORCELANA.

\begin{tabular}{|l|c|c|c|c|c|c|}
\hline \multicolumn{2}{|l|}{ Formulaciones de Porcelana } & $\mathbf{1}$ & $\mathbf{2}$ & $\mathbf{3}$ & $\mathbf{4}$ & $\mathbf{5}$ \\
\hline \multicolumn{2}{|l|}{ Porcelana Virgen (\%) } & 50.00 & 50.00 & 50.00 & 100.0 & 20.00 \\
\hline $\begin{array}{l}\text { Porcelana } \\
\text { Reciclada }(\%)\end{array}$ & Bizcochada & ---- & 50.00 & ---- & ---- & ----- \\
\cline { 2 - 7 } & Calcinada & 50.00 & ---- & 50.00 & ---- & 80.00 \\
\hline Tiempo de molienda (horas) & 16 & 8 & 11 & ---- & 9 \\
\hline $\mathbf{D}_{\mathbf{9 0}}(\boldsymbol{\mu m})$ & 12.50 & 12.68 & 21.69 & 29.99 & 24.59 \\
\hline $\mathbf{D}_{\mathbf{1 0}}(\boldsymbol{\mu m})$ & 1.08 & 1.05 & 1.33 & 1.31 & 1.33 \\
\hline $\mathbf{D}_{\mathbf{5 0}}(\boldsymbol{\mu m})$ & 4.1 & 4.0 & 6.5 & 6.6 & 7.0 \\
\hline Superficie específica $\left(\mathbf{m}^{2} / \mathbf{g}\right)$ & 2.4 & 2.5 & 1.8 & 1.8 & 1.8 \\
\hline Parámetro de pendiente $\left(\mathbf{S}_{\mathbf{w}}\right)^{*}$ & 2.41 & 2.11 & 2.37 & 1.88 & 2.02 \\
\hline Grado de compactación $\mathbf{( \% )}$ & 60 & 66 & 60 & 69 & 69 \\
\hline
\end{tabular}

\subsection{2.- CARACTERÍSTICAS DEL LIGANTE}

En todos los casos se ha utilizado el mismo ligante comercial de la casa BAYER comercialmente conocido como BAYCERAM. Este ligante está especialmente indicado para el moldeo por inyección de porcelana. La Tabla 2 muestra sus características más significativas.

TABLA 2: CARACTERÍSTICAS DEL LIGANTE.

\begin{tabular}{|c|c|}
\hline $\begin{array}{l}\text { Punto de } \\
\text { Fusión }\end{array}$ & $93-100^{\circ} \mathrm{C}$ \\
\hline $\begin{array}{l}\text { Viscosidad } \\
\left(130^{\circ} \mathrm{C}\right)\end{array}$ & 4.7 Pa s \\
\hline $\begin{array}{l}\text { Densidad } \\
(\mathrm{g} / \mathrm{cc})\end{array}$ & 0.96 \\
\hline Composición & $\begin{array}{c}\text { PE+ Poliesteramida }+ \\
\text { Polisiloxano }\end{array}$ \\
\hline
\end{tabular}

\subsection{3.- PREPARACIÓN DE LAS MEZCLAS}

El ligante y la porcelana fueron premezclados en una mezcladora tipo Doble-Sigma a $140^{\circ} \mathrm{C}$ y durante dos horas. Esta premezcla fue posteriormente mezclada y peletizada en una extrusora de doble husillo marca APV a la misma temperatura.

\section{2- Parámetros reológicos}

El equipo utilizado para el estudio reológico, es un Plastómettro de extrusión de la casa Tinus-Olsen modelo Melt-In MWLD MP 993. Este equipo funciona a esfuerzo de cizalla constante. Todas las medidas fueron realizadas en base a la norma ASTM [4]. El capilar utilizado tiene un diámetro de $2.0955 \mathrm{~mm}$ y una longitud de $8.000 \mathrm{~mm}$ siendo la relación L/D de 3.82 .

Las mezclas fueron estudiadas en un rango de temperaturas de $130-160^{\circ} \mathrm{C}$ y un rango de velocidad de cizalla de 5$2000 \mathrm{~s}^{-1}$

Utilizando la información recogida en el plastómetro, se estudiaron los siguientes parámetros reológicos: Concentración Crítica, Viscosidad, Índice de la ley de Potencias, Energía de Activación y el Índice General Reológico. A continuación se describe el significado de cada parámetro:

- Concentración Crítica en Volumen de Porcelana (CCVP): En el proceso de moldeo por inyección es crucial reducir al máximo el contenido del ligante. No solo por aspectos económicos, sino porque la etapa de eliminación es mucho más fácil y la contracción que sufre la pieza es menor.

- Viscosidad: La viscosidad es el parámetro reológico más importante en el moldeo por inyección. Un valor muy alto de la viscosidad imposibilitaría la inyección, mientras que un valor muy bajo, podría dar lugar a segregación entre la porcelana y el ligante. Este parámetro se ha estudiado en el rango de temperaturas y de velocidad de cizalla que corresponde a la etapa de inyección $\left(10-2000 \mathrm{~s}^{-1}\right)$.

La viscosidad se ha determinado mediante la siguiente expresión matemática:

$$
\eta=\frac{\sigma}{\dot{\gamma}_{w}}
$$

Donde,

$\eta$, es la viscosidad de la mezcla.

$\sigma$, es el esfuerzo de cizalla de la mezcla.

$\dot{\gamma}_{w}$, es la velocidad de cizalla en la pared.

Debido a que las mezclas tienen un comportamiento seudoplástico, es necesario realizar la corrección de Rabinowisch [5]:

$$
\dot{\gamma_{w}}=\frac{\dot{\gamma_{a}}}{4}\left(3+\frac{d \ln \dot{\gamma}_{a}}{d \ln \sigma_{w}}\right)
$$

Donde

$\dot{\gamma}_{a}$, es la velocidad de cizalla aparente.

$\sigma_{w^{\prime}}$ es el esfuerzo de cizalla en la pared.

- Índice de la Ley de Potencias (n): Las mezclas que se utilizan para el moldeo por inyección generalmente se consideran Pseudoplásticas, dicho en otras palabras, que la viscosidad disminuya con la velocidad de cizalla. El caso de la porcelana no es una excepción. Utilizando la expresión matemática propuesta por Ostwald y De Waele, [6,7], se obtiene un parámetro, $\mathrm{n}$, el cual indica la sensibilidad de la viscosidad frente a la velocidad de cizalla. 


$$
\dot{\gamma}_{a}=K \sigma^{n}
$$

Donde,

$\dot{\gamma}_{a^{\prime}}$, es la velocidad de cizalla aparente.

$\sigma$, es el esfuerzo de cizalla.

$\mathrm{K}$, es una constante.

$\mathrm{n}$, es el índice de la ley de potencias: $0<\mathrm{n}<1$.

El valor de " $\mathrm{n}$ " indica el grado de sensibilidad de la viscosidad frente a la velocidad de cizalla. Cuanto menor sea el valor de " $\mathrm{n}$ " mayor será la influencia de la velocidad de cizalla en la viscosidad. Cuanto más complicada sea la pieza que se está moldeando, más Pseudoplástica debe ser la mezcla (menor tiene que ser el valor de $\mathrm{n}$ ).

- Energía de Activación ( $E_{a}$ ): La energía de activación relaciona la viscosidad con la temperatura. Un valor alto de esta energía significa que la viscosidad tiene una fuerte dependencia con la temperatura. Esta propiedad es importante puesto que a medida que la mezcla avanza por el molde, la temperatura disminuye y la mezcla se enfría. A pesar de esa disminución de la temperatura, el material debe tener la viscosidad suficientemente baja como para seguir fluyendo.

- Índice General Reológico: $\left(\alpha_{\mathrm{STV}}\right)$ : Este índice, que engloba las variables más importantes descritas anteriormente, es una estimación del comportamiento de las mezclas en la inyección. Un valor alto de éste, indica unas buenas propiedades de flujo en la etapa de inyección.

\section{3- Pruebas de inyección}

Las pruebas de inyección se realizaron moldeando la forma de una probeta de flexión recomendada por la MPIF en una máquina de inyección ARBURG 370C 800-225. La figura 1 muestra un diagrama de la probeta inyectada.
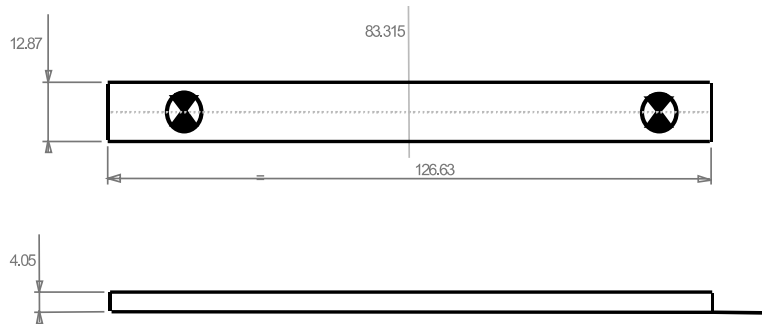

Figura 1. Diagrama de la probeta de flexión.

Las pruebas de Inyección se realizaron con objeto de:

1. Confirmar la relación entre las y propiedades reológicas y el comportamiento en el moldeo.

2. Comparar las diferentes mezclas.

3. Confirmar la inyectabilidad de las mezclas.

Los parámetros de inyección optimizados son los siguientes:

- Temperatura de Inyección $\ldots \ldots \ldots \ldots$. . . . . 150º $\mathrm{C}$

- Velocidad de Inyección . . . . . . . . . . . . . .15cc/s

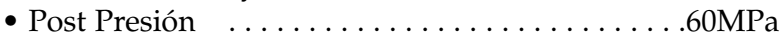

- Tiempo de Enfriamiento ...............25s

\section{3.- RESULTADOS Y DISCUSIÓN}

\section{1- Cálculo de CCVP}

Para el cálculo de la concentración crítica en volumen de porcelana se ha utilizado el modelo matemático propuesto por Maron-Pierce utilizado para mezclas altamente cargadas [8]. Este modelo ha sido utilizado en otro tipo de materiales para el moldeo por inyección de polvos [9].

$$
\eta_{\mathrm{r}}=\left(1-\frac{\Phi}{\Phi_{c}}\right)^{-2}
$$

Donde:

$\eta_{\mathrm{r}}:$ Viscosidad Relativa.

$\phi:$ Fracción volumétrica de Polvo.

$\phi_{c}$ : Fracción Crítica en Volumen de Polvo.

La viscosidad relativa se define de la siguiente manera: $\eta_{\mathrm{m}} / \eta_{0}$

$\eta_{\mathrm{m}}:$ Viscosidad de la Mezcla.

$\eta_{0}$ :Viscosidad del Ligante.

Las cinco formulaciones de porcelana se ajustan bien al modelo descrito, obteniéndose un coeficiente de regresión (R) superior a 0,90 en todos los casos. Como ejemplo se muestra el ajuste de la formulación 1 (figura 2).

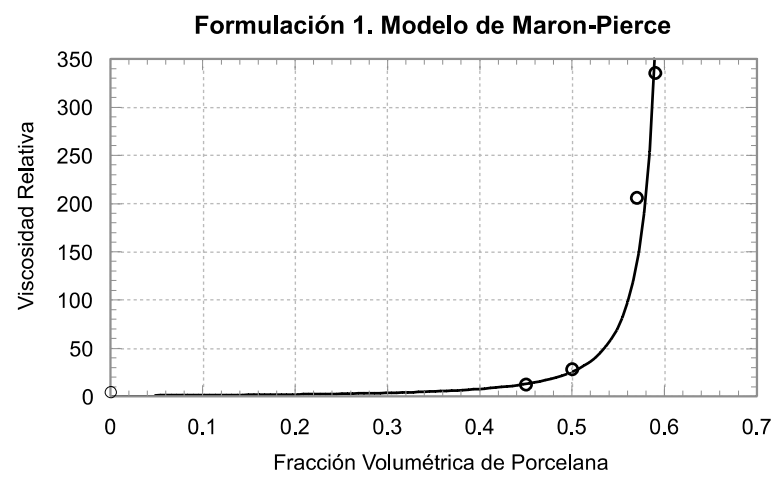

Figura 2: Ajuste de la formulación 1 al modelo de Maron-Pierce.

Los valores de las concentraciones críticas de porcelana para cada formulación se muestran en la Tabla 3.

Como se puede apreciar, la granulometría y el grado de compactación de la porcelana utilizada tienen una notable importancia en la concentración crítica en volumen de polvo. En líneas generales se puede afirmar que:

Cuanto mayor sea el grado de compactación, mayor es la CCVP.

Cuanto mayor es el tamaño medio de partícula, mayor es la CCVP.

Cuanto más ancha es la distribución de tamaños de partícula, (valores pequeños de $S_{W}$ ), mayor es la CCVP. Según se cita en la bibliografía [10, 11], el valor de $S_{w}$ debe ser menor que 2 o por el contrario mayor que 7 , para materiales en polvo destinados al proceso de moldeo por inyección.

Debido a que la mezcla ligante/porcelana puede contener heterogeneidades y con objeto de no dañar los equipos utiliza- 
dos en el mezclado y en la inyección, el contenido habitual de ligante suele ser superior al crítico calculado anteriormente. Este exceso suele ser alrededor del $5 \%$ en volumen (depende de las características del polvo, geometría de partícula, etc.). Las mezclas que se prepararon para ser inyectadas están descritas en la Tabla 3. En este caso se ha utilizado un exceso de un 5\% con respecto al modelo de Maron-Pierce de la Tabla 3, excepto para el caso de la Formulación 2 y 3 que tras ciertas pruebas y viendo los problemas de fluidez que presentaba se decidió utilizar un exceso de ligante del $8 \%$ y $6 \%$ respectivamente.

TABLA 3: CONCENTRACIONES CRÍtICAS PARA LAS FORMULACIONES DE PORCELANA ESTUDIADAS Y MEZCLAS PREPARADAS.

\begin{tabular}{|c|c|c|c|}
\hline PORCELANA & MEZCLA & CCVP & $\begin{array}{c}\text { \% vol. (\%wt) } \\
\text { PORCELANA }\end{array}$ \\
\hline $\mathbf{1}$ & M1 & $\mathbf{0 . 6 2}$ & $\mathbf{5 7 \% ( 7 7 \% )}$ \\
\hline $\mathbf{2}$ & M2 & $\mathbf{0 . 6 4}$ & $\mathbf{5 6 \% ( 7 6 \% )}$ \\
\hline 3 & M3 & $\mathbf{0 . 6 9}$ & $\mathbf{6 3 \%}(\mathbf{8 1 \%})$ \\
\hline 4 & M4 & $\mathbf{0 . 6 8}$ & $\mathbf{6 3 \% ( 8 1 \% )}$ \\
\hline 5 & M5 & $\mathbf{0 . 7 2}$ & $\mathbf{6 7 \% ( 8 4 \% )}$ \\
\hline
\end{tabular}

\section{2- Medidas de viscosidad}

La viscosidad de cada mezcla se ha determinado a temperaturas y velocidades de cizalla en las que se efectúa la inyección. Como resumen la Tabla 4 muestra la viscosidad de cada mezcla a la temperatura de inyección $\left(150^{\circ} \mathrm{C}\right)$ y una velocidad de cizalla de $100 \mathrm{~s}^{-1}$.

TABLA 4: RESUMEN DE LOS VALORES DE LAS CARACTERÍSTICAS REOLÓGICAS ESTUDIADAS.

\begin{tabular}{|c|c|c|c|c|}
\hline MEZCLA & $\begin{array}{c}\eta^{*} \\
(\mathbf{P a} \text { s) }\end{array}$ & $\mathbf{n}$ & $\begin{array}{c}\text { ENERGIA DE } \\
\text { ACTIVACION(KJ/mol) }\end{array}$ & $\begin{array}{c}\text { INDICE GENERAL } \\
\text { REOLOGICO: } \boldsymbol{\alpha}_{\text {sTv }}\end{array}$ \\
\hline M1 & 650 & 0,44 & 29 & 55 \\
\hline M2 & 435 & 0,51 & 26 & 53 \\
\hline M3 & 476 & 0,48 & 28 & 52 \\
\hline M4 & 693 & 0,41 & 22 & 74 \\
\hline M5 & 724 & 0,49 & 31 & 35 \\
\hline
\end{tabular}

Cabe destacar que todas las mezclas tienen una viscosidad menor que $1000 \mathrm{~Pa}$ s. Este valor arbitrario se toma como referencia para predecir si una mezcla se puede inyectar o no [9]. Se puede observar que las mezclas M2 y M3 son las que menor viscosidad tienen y por lo tanto más fácilmente podrán ser inyectadas, no obstante siguiendo el criterio mencionado anteriormente todas las mezclas serían adecuadas para ser inyectadas.

\section{3- Influencia de la velocidad de cizalla en la viscosidad: "n"}

Durante el moldeo por inyección es deseable tener un fluido Pseudoplástico, especialmente cuando se están moldeando piezas muy complicadas. En la figura 3 se muestran las gráficas a partir las cuales se calcula el valor del índice.

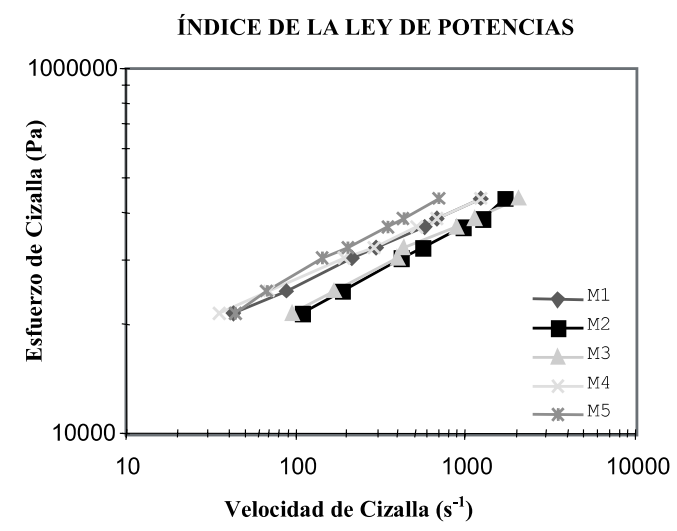

Figura 3: $\log$ Esfuerzo de cizalla vs. $\log$ Velocidad de cizalla a $150^{\circ} \mathrm{C}$.

Los valores de $\mathrm{n}$ (calculados en un rango de velocidad de cizalla entre $50 \mathrm{~s}^{-1}$ y $1700 \mathrm{~s}^{-1}$ ) se calculan a partir de la pendiente de las curvas de la figura 3 . Cuanto más bajo sea el valor de $n$, mayor es la influencia de la velocidad de cizalla en la viscosidad.

Estudiando la variación del índice de la Ley de Potencias con la concentración de sólidos, se ha observado que éste disminuye al aumentar la concentración de porcelana en la mezcla (un ejemplo de este fenómeno se observa en la figura 4). Esto es lo esperado si se tiene en cuenta que la naturaleza del ligante puro es totalmente Newtoniana. A medida que la mezcla se hace más concentrada, el carácter Pseudoplástico aumenta.

\section{INDICE DE LA LEY DE POTENCIAS EN FUNCION DE LA CONCENTRACION DE PORCELANA EN LA MEZCLA}

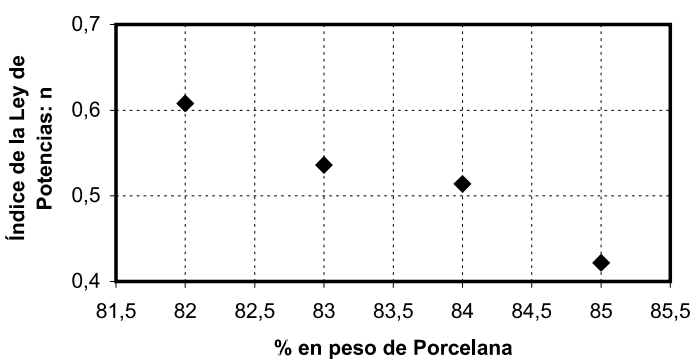

Figura 4: Variación del índice de la Ley de Potencias en función de la concentración de porcelana en la mezcla para la formulación 5.

La mezcla M4 es la más sensible a la velocidad de cizalla, mientras que la mezcla M2 es la menos sensible (Tabla 4). Esto puede deberse a la mayor cantidad de ligante en la mezcla M2. Desde el punto de vista de la Pseudoplasticidad, la mezcla M4 es la más indicada para la inyección.

\section{4- Influencia de la temperatura en el comportamiento reológico}

La temperatura desempeña un papel de primer orden en el moldeo por inyección. Por eso es importante tener un detallado conocimiento de la dependencia de la viscosidad frente a la temperatura. Este comportamiento se puede expresar 
mediante una expresión matemática tipo Arrhenius:

$$
\eta(T)=\eta\left(T_{0}\right) \exp \left(\frac{E_{a}}{R}\left[\frac{1}{T}-\frac{1}{T_{0}}\right]\right)
$$

Donde,

$E_{a^{\prime}}$ es la energía de activación de flujo.

$\mathrm{R}$, es la constante universal de los gases.

$\mathrm{T}$, es la temperatura.

$\eta\left(T_{0}\right)$, es la viscosidad a la temperatura de referencia $T_{0}$.

La energía de activación se obtiene de la pendiente de la curva de la representación gráfica de $\operatorname{lnh}$ frente a $1 / \mathrm{T}$. Esta gráfica se ha realizado a una velocidad de cizalla constante de $100 \mathrm{~s}^{-1}$. La tabla 4 muestra los valores de energía de activación para las cinco mezclas. Como puede observarse, los valores son muy similares en todas las mezclas, y a su vez muy similares a la energía de activación de ligante puro $\left(\mathrm{E}_{\mathrm{a}}=32 \mathrm{KJ} / \mathrm{mol}\right)$. Esta ausencia del efecto de la concentración de sólidos en el valor de $\mathrm{E}_{a^{\prime}}$ es típica en la reología de polímeros altamente cargados [12]. La energía de activación depende principalmente del tipo de ligante que se esté utilizando, y no tanto del tipo de polvo o concentración de éste. No obstante la mezcla M4 es ligeramente más favorable desde este punto de vista, mientras la mezcla M5 sería la menos favorable

\section{5- Índice general reológico}

Con objeto de establecer un índice que englobe los parámetros reológicos más importantes, se ha utilizado el modelo propuesto por Weir para polímeros [13].

$$
\alpha_{S T V}=\frac{1}{\eta\left(T_{0}\right)} \frac{|n-1|}{E_{a} / R}
$$

Donde,

$\eta\left(T_{0}\right)$, es la viscosidad a la temperatura de referencia $T_{0}$. $\mathrm{E}_{\mathrm{a}^{\prime}}$ es la energía de activación.

$\mathrm{n}$ es el índice de la ley de potencias.

$\mathrm{R}$, es la constante universal de los gases.

Cuanto más alto sea el valor de $\alpha_{\mathrm{STV}}$, mejores serán las propiedades reológicas para el moldeo por inyección. Los valores de este índice calculados a $150^{\circ} \mathrm{C}$ y velocidad de cizalla de $100 \mathrm{~s}^{-1}$ se encuentra en la tabla 4 (los valores han sido multiplicados por $10^{6}$ ).

Como se puede observar la mezcla M4 tiene el mayor valor de $\alpha_{\mathrm{STV}}$, por lo que es la mezcla más apropiada para la inyección. Las mezclas M1, M2 y M3 tienen un valor del índice muy similar, mientras que la mezcla M5 tiene el valor más bajo del índice por lo que es la peor candidata para el moldeo por inyección desde el punto de vista reológico.

\section{6- Moldeo por inyección}

Todas las probetas moldeadas presentan una apariencia visual excelente, no se observan defectos como rechupes, rebabas, falta de llenado, etc.(figura 5).

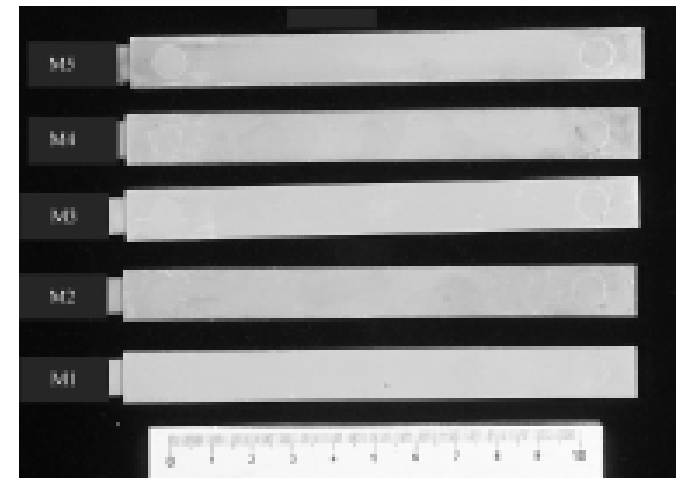

Figura 5: Probetas inyectadas.

Sin embargo cabe destacar que la mezcla M5, ha dado resultados adversos en lo que respecta al molde. Debido a las condiciones reológicas de esta mezcla y la geometría de las partículas, el molde ha resultado dañado, especialmente las zonas más estrechas (entrada a la cavidad). En la figura 6 se observa el desgaste sufrido en la entrada a la cavidad.

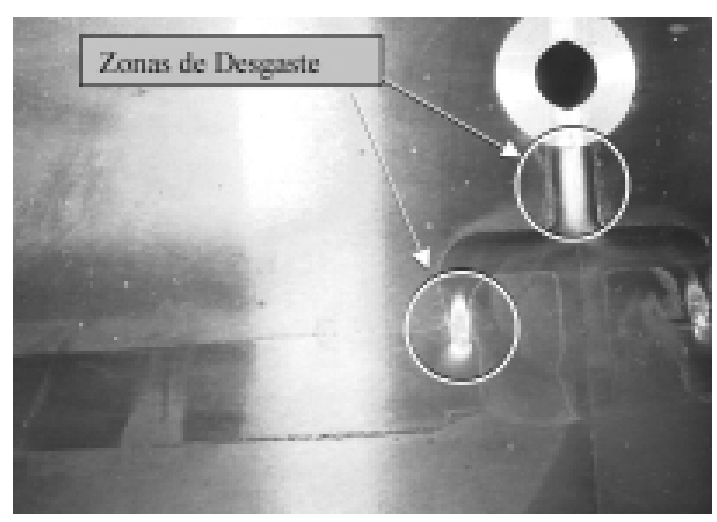

Figura 6: Molde Dañado.

Como consecuencia de esta inyección defectuosa, se puede considerar que la mezcla M5 no es una buena candidata para el moldeo por inyección. Esto confirma la validad del índice general reológico el cual concede el menor valor a la mezcla M5 $\left(\alpha_{\mathrm{STV}}=35\right)$. Como un primer resultado preliminar se podría concluir diciendo que las mezclas de porcelana para la inyección deben tener un índice mayor que 35 para que el moldeo se produzca en buenas condiciones.

$\mathrm{Al}$ tiempo que este artículo estaba siendo escrito, se ha continuado con la investigación en la etapa de extracción del ligante y sinterización. Un resultado preliminar arroja los resultados que la mezcla M4 además de ser la mejor en la etapa del moldeo, también lo son en la etapa de extracción y sinterización. Con la mezcla M3 también se obtiene resultados satisfactorios en estas etapas. Sin embargo no fue posible extraer el ligante de las mezclas M1 y M2. Así pues parece que la reología influye en la calidad de las probetas obtenidas, aunque existen otros parámetros (grado de compactación, geometría de partícula, etc.) que ejercen su influencia en etapas posteriores a la de moldeo; por lo que un buen comportamiento reológico es necesario para obtener una buena pieza, pero no es suficiente para completar con éxito el proceso de 
moldeo por inyección. En la figura 7 se muestra el resultado de una de las pruebas de eliminación y sinterización con una de las formulaciones (mezcla M3). Corresponde a una geometría tipo jarra obtenida con un $50 \%$ de porcelana reciclada.

\section{4.- CONCLUSIONES}

Se ha comprobado satisfactoriamente que es posible la inyección de porcelana reciclada.

Las características granulométricas del polvo son muy importantes puesto que utilizando el mismo ligante, aquellas dictan el comportamiento reológico.

Se ha demostrado que es posible utilizar el Índice General Reológico para evaluar las propiedades de flujo de una mezcla.

Las mezclas M1, M2, M3 y M4 son aptas para el moldeo por inyección, si bien la mejor candidata según el índice general reológico es la mezcla M4. Unos resultados preliminares de eliminación y sinterización dan como mejores mezclas a las mezclas M3 y M4.

Aunque las propiedades reológicas expresadas a través del índice General Reológico influyen en la calidad de las piezas, existen otros parámetros tales como grado de compactación, distribución de tamaños de partícula, geometría de partícula, etc. que condicionan el éxito de la obtención de piezas en etapas posteriores (eliminación y sinterización) a la del moldeo.

\section{5.- AGRADECIMIENTOS}

Los autores desearían agradecer a la Comunidad Económica Europea por la financiación del Programa BRITEEURAM (Proyecto BE95-146), a KPCL, Soarmoldes y Porcelanas del Bidasoa por su apoyo y contribución con conocimientos de la porcelana.

\section{6.- REFERENCIAS}

1. W.Ryan and C.Radford, "Whitewares: Production, Testing and Quality Control", Pergamon Press (1987).

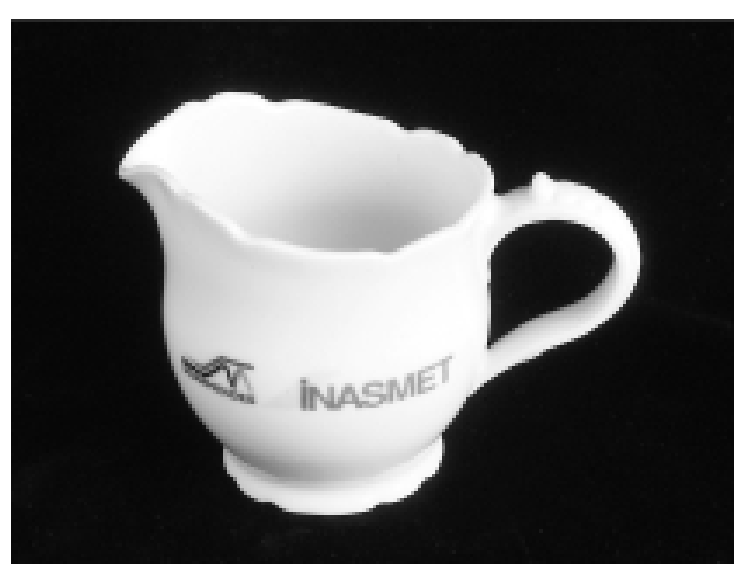

Figura 7: Jara sinterizada con la mezcla M3.

2. Engineered Materials Handbook: Ceramic and Glasses. Vol. 4 ASM International, The Materials Information Society (1991)

3. R.M.German, "Particle Packing Characteristics". Metal Powder Industries Federation, Princeton, New Jersey, USA (1989)

4. "Standard Test Method for Flow Rates of Thermoplastics by Extrusion Plastometer" Annual Book of ASTM Standards, ASTM, Vol. 08.01, , p p 394402 (1993)

5. B. Rabinowitsch,, Z. Physik. Chem., A(145), 1 (1929)

6. W. Ostwald, Kolloid-Z, 36, 99 (1925)

7. A. De Waale, Oil and Color Chem. Assoc. J., 6. 33 (1923)

8. S.H. Maron, P.E. Pierce, J.Colloid Sci 11, 80 (/1956)

9. R.M. German and Animesh Bose, "Injection Molding of Metals and Ceramics", Metal Powders Industry Federation (1997)

10. R.G. Iaccocca, "A critical assessment of characterisation test needed to support PIM component fabrication" Reviews in Particulate Materials, Vol. 2, Pg. 314 (1994)

11. K.F.Hens, R.M.German, "Advanced processing of Advanced materials via PIM", Advances in Powder Metallurgy \& Particulate Material, Vol. 5, pg. 5773, (1993)

12. G.Marin in A.A.Collyer and D.W.Clegg, "Rheological Measurements", Elsevier Applied Science, London (1988)

13. F.E.Weir, M.E.Doyle and D.G.Norton, "Mouldability of Plastic Based on Melt Rheology" S.P.E. Transactions 3 pp. 32-36 (1963) 